\title{
Corela
}

Cognition, représentation, langage

HS-30 | 2020

Phonétique, littérature et enseignement du FLE : théories et recherches

\section{Enseignement/apprentissage du FLES aux EFIV : propositions didactiques de l'utilisation de comptines à gestes pour la perception et la production des phonèmes et graphèmes du français}

Diane Caussade, Sandra Cornaz et Marina Yezhkova

\section{OpenEdition \\ Journals}

Édition électronique

URL : http://journals.openedition.org/corela/10596

DOI : 10.4000/corela.10596

ISSN : 1638-573X

Éditeur

Cercle linguistique du Centre et de l'Ouest - CerLICO

Référence électronique

Diane Caussade, Sandra Cornaz et Marina Yezhkova, «Enseignement/apprentissage du FLES aux EFIV : propositions didactiques de l'utilisation de comptines à gestes pour la perception et la production des phonèmes et graphèmes du français », Corela [En ligne], HS-30 | 2020, mis en ligne le 04 mai 2020, consulté le 22 mai 2020. URL : http://journals.openedition.org/corela/10596 ; DOI : https://doi.org/10.4000/corela.10596

Ce document a été généré automatiquement le 22 mai 2020.

\section{(i) (1)(2)}

Corela - cognition, représentation, langage est mis à disposition selon les termes de la licence Creative Commons Attribution - Pas d'Utilisation Commerciale - Partage dans les Mêmes Conditions 4.0 International. 


\title{
Enseignement/apprentissage du FLES aux EFIV : propositions didactiques de l'utilisation de comptines à gestes pour la perception et la production des phonèmes et graphèmes $d u$ français
}

\author{
Diane Caussade, Sandra Cornaz et Marina Yezhkova
}

\section{Introduction}

1 En France, les diverses communautés désignées par l'appellation administrative « gens du voyage », sont pour la grande majorité de nationalité française depuis plusieurs générations (de Gila-Kochanowski, 1994 ; Thibault, 1999 ; Bader, 2007 ; Welschinger, 2007 ; Welschinger, 2013). Pour autant, le français est une langue-culture seconde, voire étrangère, pour les enfants issus de familles itinérantes et de voyageurs (EFIV). En effet, les « gens du voyage » se répartissent en plusieurs langues-cultures distinctes pour qui le français n'est pas la langue maternelle (LM). Devant l'important taux d'analphabétisme, ainsi que le faible taux de scolarisation des EFIV, leur insertion socioculturelle semble primordiale (Service Gens du voyage, 2017). Les langues-cultures des «gens du voyage" étant principalement de transmission orale (de GilaKochanowski, 1994 ; Clanet, 2007), l'utilisation de comptines à gestes comme support de l'enseignement/apprentissage du français langue étrangère et seconde (FLES) pourrait alors être pertinent. L'utilisation des gestes manuels auprès d'EFIV est d'autant plus à propos que le non-verbal fait partie intégrante du langage (Colletta, 2004; McNeill, 2000) et favorise la compréhension (Hubbard et al., 2002 ; Tellier, 2009) et la production langagière (McNeill, 1992 ; Feyereisen et Havard, 1999). Des travaux ont trouvé que le geste était aussi un support pour l'enseignement/apprentissage de la prosodie et de la 
phonologie (Borel-Maisonny, 1986; Miranda, 2012). Au vu de ces éléments, des comptines à gestes ont été créées afin de permettre l'enseignement/apprentissage d'une part de la perception et de la production de phonèmes du français, d'autre part de la correspondance entre phonèmes et graphèmes à un public d'EFIV. Ces supports pédagogiques ont été testés par l'Association Ballade auprès d'EFIV. Leurs effets ont ensuite été analysés afin de permettre une réévaluation des propositions didactiques.

Cette recherche-action ${ }^{1}$ se propose, dans une première partie, de présenter les spécificités des interventions éducatives auprès d'EFIV, notamment liées à leurs langues-cultures et à leur scolarisation. L'intérêt de l'utilisation de comptines à gestes pour l'enseignement/apprentissage du FLES aux EFIV sera expliqué. Dans une seconde partie, la mise en place de l'action pédagogique sur support de comptines à gestes auprès d'EFIV sera explicitée. De plus, les effets de cette action pédagogique sur l'enseignement/apprentissage du FLES à des EFIV - en particulier sur le plan de la perception et de la production des phonèmes, et de la correspondance entre phonèmes et graphèmes-seront analysés en vue de permettre une amélioration des interventions socioculturelles auprès de ce public.

\section{Spécificités des interventions éducatives auprès d'EFIV}

3 Depuis 2015, le Service Gens du voyage de l'Eurométropole de Strasbourg travaille à la définition et à la mise en place d'un projet social pour répondre aux besoins des habitants des aires d'accueil des "gens du voyage", et pour accompagner les associations intervenant sur ces aires (Service Gens du voyage, 2017). Ce projet expérimental inédit est un processus d'élaboration continu permettant un ajustement permanent aux besoins spécifiques de ces communautés. L'association strasbourgeoise Ballade travaille en partenariat avec le Service Gens du Voyage de l'Eurométropole de Strasbourg afin de soutenir l'animation de la vie sociale de proximité par le biais d'actions musicales sur l'aire d'accueil des « gens du voyage » de Geispolsheim.

\section{1. Langues-cultures des " gens du voyage »}

4 Nous rapportons ici un ensemble d'éléments qui méritent d'être considérés par des personnes intervenant auprès de "gens du voyage ", et plus particulièrement d'EFIV.

5 Le terme EFIV est utilisé par l'Éducation Nationale pour désigner les enfants des « gens $\mathrm{du}$ voyage ». L'appellation "gens du voyage» correspond à une catégorie administrative destinée, depuis 1969, aux citoyens français dont le mode d'habitat mobile est à la base de l'organisation sociale, même si tous ne sont pas ou plus itinérants (Aubin, 2008; Pluchon, 2009). Quel que soit le degré spatiotemporel de mobilité, les « gens du voyage » sont souvent ancrés dans un territoire (Service Gens du voyage, 2017). Les causes de la mobilité peuvent être à la fois (ibid.) :

- Fonctionnelles, permettant l'organisation socioprofessionnelle ;

- Structurelles, autorisant l'adaptabilité aux contraintes socioéconomiques ;

- Culturelles, permettant la participation aux évènements familiaux et religieux.

6 Le sens de l'appellation "gens du voyage » a progressivement glissé pour désigner un groupe ethnique qui n'en est en fait pas un, puisqu'il comprend réellement plusieurs 
langues-cultures. Ainsi, les «gens du voyage » comprennent plusieurs communautés, notamment (de Gila-Kochanowski, 1994 ; Thibault, 1999) : désignent souvent par le terme de «Voyageurs » (Thibault, 1999). Les « Voyageurs » se définissent en distinction aux non-voyageurs ou gadjé, cette distinction révèle leur peur de l'acculturation (ibid.). Comme expliqué plus avant, les «Voyageurs»se caractérisent par leur mode de vie nomade, mais aussi par leurs modes de vie communautaires où la famille a un sens élargi de clan, lignage (Service Gens du voyage, 2017). La hiérarchie des « gens du voyage » est également différente de celle des gadjé : les "gens du voyage" fonctionnent de manière horizontale, alors que les gadjé fonctionnent de manière verticale (Liégeois, 1994). Ainsi, chez les " gens du voyage ", il n'y a pas de différence entre l'enfance et l'âge adulte (ibid.). L'apprentissage des enfants est fait de manière active et indépendante sans contraintes horaires strictes, ces derniers disposent d'une grande autonomie avec un contrôle global, par le groupe (ibid.).

15 Actuellement, l'Eurométropole de Strasbourg gère huit aires pérennes d'accueil des "gens du voyage » (Service Gens du voyage, 2017). L'aire d'accueil des "gens du voyage » de Geispolsheim comprend trente-sept places de stationnement (ibid.). Cette aire accueille des familles de langues-cultures différentes ${ }^{3}$. Le Service Gens du voyage de l'Eurométropole de Strasbourg rapporte les problématiques de vivre-ensemble sur ces aires dues aux différences langagières et culturelles des communautés accueillies sur ces aires (ibid.). Au vu de ces éléments, les spécificités langagières et culturelles des "gens du voyage " sont une donnée importante à prendre en compte dans la mise en place d'actions auprès de ces publics.

\section{2. Scolarisation des EFIV}

16 La scolarisation des EFIV est faite en lien avec le Centre Académique pour la Scolarisation des élèves allophones Nouvellement Arrivés et des élèves issus de familles itinérantes et de Voyageurs (CASNAV). La circulaire $\mathrm{n}^{\circ} 2012-142 \mathrm{du} 02$ octobre 2012 définit les EFIV comme «issus de familles itinérantes et de familles sédentarisées depuis peu, ayant un mode de relation discontinu à l'école ». Bien que le code de 
l'Éducation spécifie que « le statut et le mode d'habitat des familles [...] ne peut être une cause de refus d'inscription d'un enfant soumis à l'obligation scolaire", le taux de scolarisation effective des EFIV reste faible (Service Gens du voyage, 2017). La distinction entre scolarisation effective et inscription dans un établissement scolaire est de mise. La durée de scolarisation effective des EFIV est variable (quelques jours parfois) et généralement inférieure à celle du séjour sur l'aire d'accueil. Il faut préciser que cette dernière est fréquemment d'une durée inférieure à celle de l'année scolaire (ibid.). De plus, le taux d'absentéisme des EFIV est également important. Les EFIV ayant des problématiques différentes d'autres élèves, ils nécessitent une attention particulière de la part des enseignants, qui n'ont pas ou peu été sensibilisés et formés aux besoins spécifiques de ces publics, et qui ne disposent pas de moyens supplémentaires pour les accompagner (ibid.). Ceci peut entraîner un décrochage scolaire des EFIV. De plus, les absences des EFIV sont souvent justifiées par leurs parents pour des motifs de convenance personnelle (ibid.). En outre, certains EFIV sont inscrits au Centre national d'enseignement à distance (CNED), ce qui ne signifie pas nécessairement leur scolarisation effective (ibid.). Et même si la scolarisation d'EFIV au CNED est effective, ceux-ci se retrouvent isolés dans leurs apprentissages car, pour la plupart, leurs parents sont dans l'impossibilité de les accompagner dans leur travail scolaire (Clanet, 2007).

Dans l'Eurométropole de Strasbourg, entre 30 et $80 \%$ des enfants, selon les aires, sont inscrits dans un établissement scolaire (Service Gens du voyage, 2017). Le taux d'inscription varie également selon les niveaux du système éducatif :

- $32 \%$ à l'école maternelle ;

- $82 \%$ à l'école élémentaire ;

- $19 \%$ au collège.

De plus, la durée de scolarisation est variable et généralement inférieure à la durée du séjour sur l'aire d'accueil, qui elle-même ne correspond souvent pas à la durée de l'année scolaire (ibid.).

19 La question de l'analphabétisme et de l'illettrisme des EFIV peut notamment s'expliquer par les facteurs suivants :

- L'illettrisme, voire l'analphabétisme, des « gens du voyage » non-scolarisés enfants (ibid.) ;

- La communication et la transmission orale de leurs LM (de Gila-Kochanowski, 1994 ; Clanet, 2007) ;

- La peur d'une acculturation, de préjugés et de discriminations de la société française (Martin, 2011 ; Service Gens du voyage, 2017);

-L'éloignement géographique et le manque d'accès aux transports en commun et scolaires (Service Gens du voyage, 2017).

Une politique sociale d'inclusion des "gens du voyage » par la création et/ou le renforcement de liens sociaux avec les «non-voyageurs" serait un moyen de lutter contre leur isolement. L'intervention de l'Association Ballade sur les aires s'inscrit dans cet objectif. 


\section{3. Intérêt de l'utilisation de comptines à gestes pour l'enseignement/apprentissage du FLES aux EFIV}

Dans la mesure où la LM des " gens du voyage » est orale (de Gila-Kochanowski, 1994 ; Clanet, 2007) et le français une langue seconde, voire étrangère, pour les EFIV (ibid.), l'utilisation de comptines à gestes pour l'enseignement/apprentissage du FLES aux EFIV paraît intéressante à explorer. Cet outil nous apparait d'autant plus adéquat qu'il permet de travailler les modalités à la fois verbale et non-verbale du langage.

En effet, le langage est multimodal (McNeill, 1992 ; McNeill et al., 2000 ; Colletta, 2004). Le langage verbal correspond à la parole et est composé du langage segmental - soit la chaîne de phonèmes qui forme l'énoncé - et suprasegmental - c'est-à-dire les traits prosodiques tels que l'intonation, l'accentuation, le rythme, etc. - (Saussure, 1971). Le langage non-verbal comprend, quant à lui, les gestes dits communicatifs: gestes manuels, expressions faciales, regards et conduites proxémiques (ex. : Brin et al., 2011). L'utilisation des gestes communicatifs ${ }^{2}$ pour l'enseignement/apprentissage du FLES est d'autant plus pertinente que ceux-ci feraient partie intégrante du développement du langage (pour une revue $c f$. Colletta, 2004). Nombre d'études ont également montré combien les gestes communicatifs sont étroitement liés à la compréhension et à la production des langues maternelles, étrangères et secondes (ex.: McNeill, 1992; McNeill et al., 2000 ; Hubbard et al., 2002 ; Tellier, 2006 ; Feyereisen et al., 2007 ; Tellier, 2009). La perception des gestes co-verbaux faciliterait, entre autres, la mémorisation d'une langue étrangère (Allen, 1995). De plus, la perception conjointe des gestes coverbaux et oro-faciaux faciliterait la compréhension des langues secondes (Sueyoshi et Hardison, 2005); ce qui pourrait être expliqué par le fait que gestes manuels et orofaciaux feraient partie d'un même système moteur, qui serait en place très tôt dans le développement (Iverson et Fagan, 2004).

L'utilisation des gestes manuels dans l'enseignement/apprentissage des langues étrangères et secondes paraît de ce fait pertinente. Les gestes manuels sont d'ailleurs utilisés dans diverses méthodes et activités d'enseignement/apprentissage, telles que verbo-tonale (Guberina, 1965; pour une revue cf. Renard, 2002), Montessori (Montessori, 1958) ou encore « ritmimots» (Llorca, 2008). Dunoyer de Segonzac (1991) utilise par exemple le geste graphique dans sa méthode de la Dynamique Naturelle de la Parole (DNP), d'ailleurs inspirée de la méthode verbo-tonale de Guberina (1965). Dans ces deux méthodes, les macromouvements produits au niveau gestuel sont utilisés pour faciliter les micromouvements oro-faciaux. Bara, Gentaz et Colé (2005) ont proposé, quant à eux, d'utiliser le geste haptique dans le cadre de l'enseignement/apprentissage de la lecture. Les gestes, mais aussi les signes, peuvent également être exploités pour l'enseignement/apprentissage lexical, comme l'ont expérimenté Caussade, Cornaz et Tisserand (2014).

Par conséquent, l'utilisation de comptines à gestes semble intéressante pour l'enseignement/apprentissage du FLE. Les comptines à gestes ont ceci de particulier qu'elles associent parole et gestes manuels. De plus, les comptines à gestes font partie intégrante du folklore enfantin (Arleo et Delalande, 1995). Plusieurs types de comptines à jeux existent, tels que les "jeux de nourrice ", les "comptines d'élimination » (i.e. « méta-jeux » ou « ploufs »), et les « tape-mains » (ibid.). L'utilisation de ces divers types 
de comptines à gestes dans l'enseignement/apprentissage des langues étrangères et secondes est un moyen d'aborder de nombreux types de gestes différents :

- Pantomimiques, ex. : dans la chanson «Dans sa maison un grand cerf», plusieurs paroles sont mimées ;

- Déictiques, ex. : dans la comptine d'élimination « Si tu ne l'as pas, c'est toi le roi », le geste de pointage est utilisé pour désigner les différents participants ;

- Emblématiques, ex. : dans le jeu de nourrice «Un et un, deux », les gestes emblématiques français des chiffres sont produits ;

-Rythmiques, ex.: dans le tape-mains «Trois petits chats», les gestes accompagnent rythmiquement la parole.

Il est également possible d'ajouter des gestes manuels à des supports oraux n'en possédant pas a priori. Les types de gestes peuvent alors être choisis selon l'objectif pédagogique.

Ainsi, des gestes pantomimiques peuvent été utilisés pour faciliter l'accès au sens d'une chanson et éviter le recours à la traduction ${ }^{3}$, pour permettre une meilleure mémorisation, pour palier d'éventuels troubles attentionnels et apporter un aspect ludique à l'enseignement/apprentissage. Pour les besoins didactiques, il est aussi envisageable de créer une comptine artificielle plutôt que de baser le lexique gestuel sur du matériel authentique quand celui-ci ne permet pas de répondre facilement à l'objectif pédagogique cible (Cornaz, 2014). Dans un contexte de lutte contre l'illettrisme et l'analphabétisme des EFIV, la création de comptines à gestes pour l'enseignement/apprentissage de la perception des phonèmes et des graphèmes semble ainsi adéquate.

\section{Mise en place et analyse de l'action pédagogique}

L'Association Ballade intervient auprès d'EFIV dans l'aire d'accueil des "gens du voyage» de Geispolsheim depuis l'année scolaire 2017-2018. Ces interventions consistent en des ateliers hebdomadaires de musiques traditionnelles. La pédagogie de l'Association Ballade est non-formelle, interactive et fondée sur une pratique instrumentale à l'oreille. Le répertoire utilisé est composé de diverses musiques traditionnelles qui dépendent des spécificités et besoins des publics cibles, et qui permettent de valoriser leurs langues-cultures d'origine et de créer un répertoire commun sur les plans musical, culturel et langagier.

\section{1. Méthodologie}

Les interventions de l'Association Ballade auprès d'EFIV sur l'aire d'accueil des " gens du voyage " de Geispolsheim consistent en l'enseignement de trois instruments: percussions (darbouka et cajon), guitare et chant. Chaque EFIV peut choisir le type et le nombre d'instruments qu'il souhaite pratiquer. Les EFIV n'ont aucune obligation de venir ni de rester aux ateliers. Selon les semaines et les moments de l'atelier, cinq à vingt EFIV y participent.

Cet article s'intéresse plus particulièrement à l'atelier de chant dans lequel a pris place cette recherche-action sur l'enseignement/apprentissage des phonèmes et graphèmes du FLES par le biais de comptines à gestes et de gestes manuels. Chaque atelier de chant 
dure deux heures et accueille tous les EFIV qui souhaitent y participer. Les EFIV participant aux ateliers de chant sont majoritairement de sexe féminin. Comme souvent les grandes sœurs viennent avec leur(s) petite(s) sœur(s) car elles en sont responsables, les participants à l'atelier de chant sont âgés de 3 à 15 ans.

Lors de la première intervention de l'Association Ballade dans l'aire d'accueil des « gens du voyage » de Geispolsheim, certains EFIV ont demandé à pouvoir dessiner aux musiciens intervenants de l'Association Ballade. Un EFIV de huit ans a alors dessiné la darbouka d'un des musiciens intervenants et écrit le nom de la marque de cette darbouka qui figurait dessus ( $c f$. Figure 1). Les musiciens intervenants ont alors remarqué le taux important d'illettrisme, voire d'analphabétisme, des EFIV, mais aussi leur envie - exprimée verbalement - d'apprendre à lire et à écrire. Ainsi, un enfant de sept ans a préféré « écrire » que dessiner, bien qu'il ne sache visiblement pas écrire (cf. Figure 2).

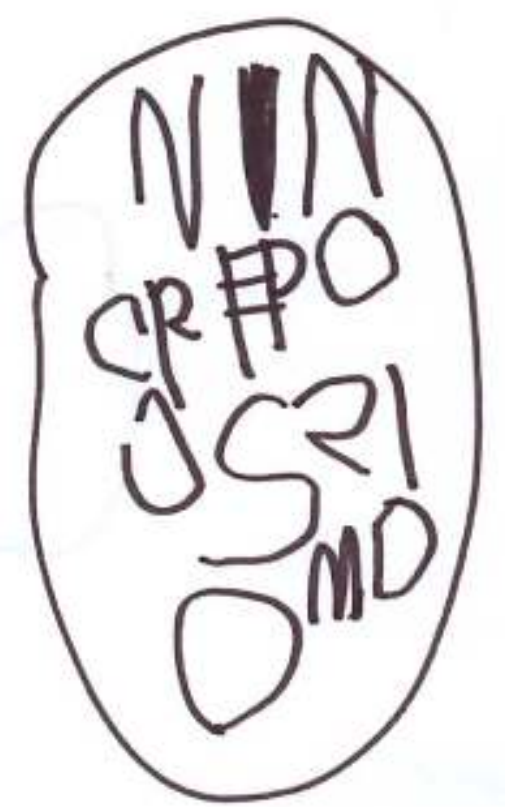

Figure 1 : dessin d'une darbouka par un EFIV de huit ans 


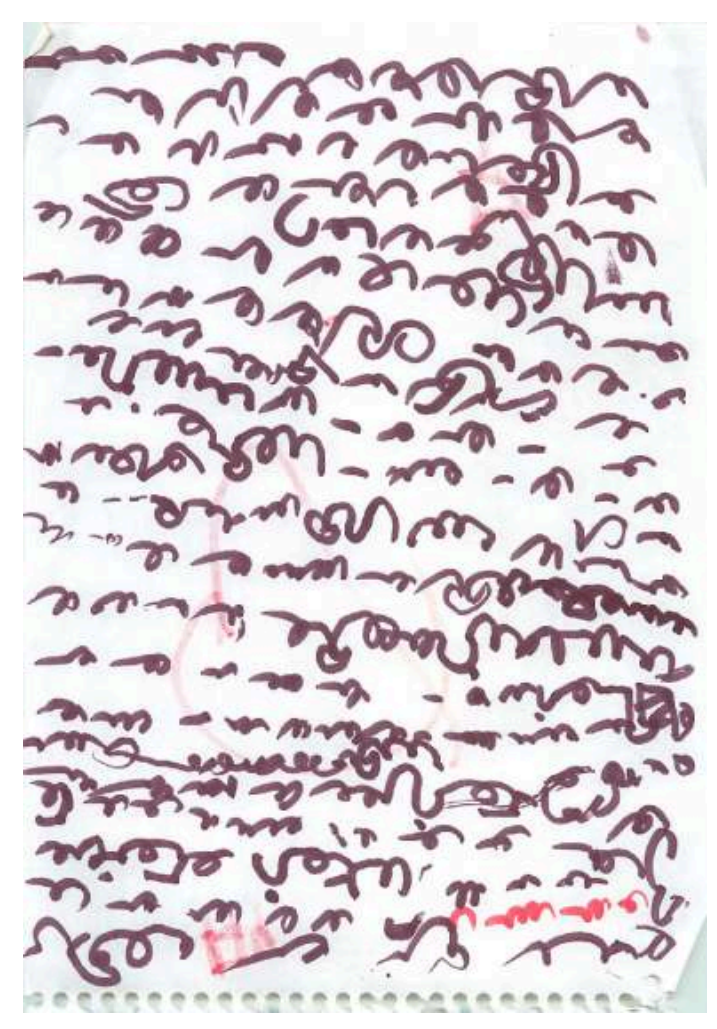

Figure 2 : page d'écriture d'un EFIV de sept ans

31 Les musiciens intervenants ont également observé que les EFIV ne savaient pas dessiner. Apparemment ces derniers n'ont d'ailleurs pas de crayons, ni de feutres, ni de feuilles chez eux. La Figure 3 montre à titre illustratif un sapin dessiné par un EFIV non-scolarisé âgé de 8 ans résidant sur l'aire d'accueil des "gens du voyage » de Geispolsheim. La Figure 4 montre, à titre comparatif, des sapins dessinés par deux enfants scolarisés âgés de 7 et 8 ans de l'espace d'accompagnement et d'insertion de familles Roms de Cronenbourg.

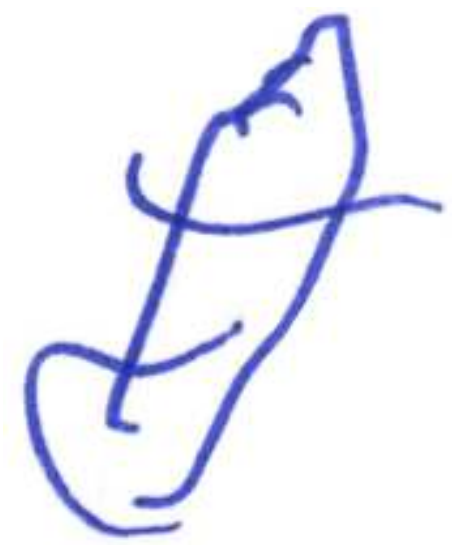

Figure 3 : dessin d'un sapin par un EFIV de 8 ans 


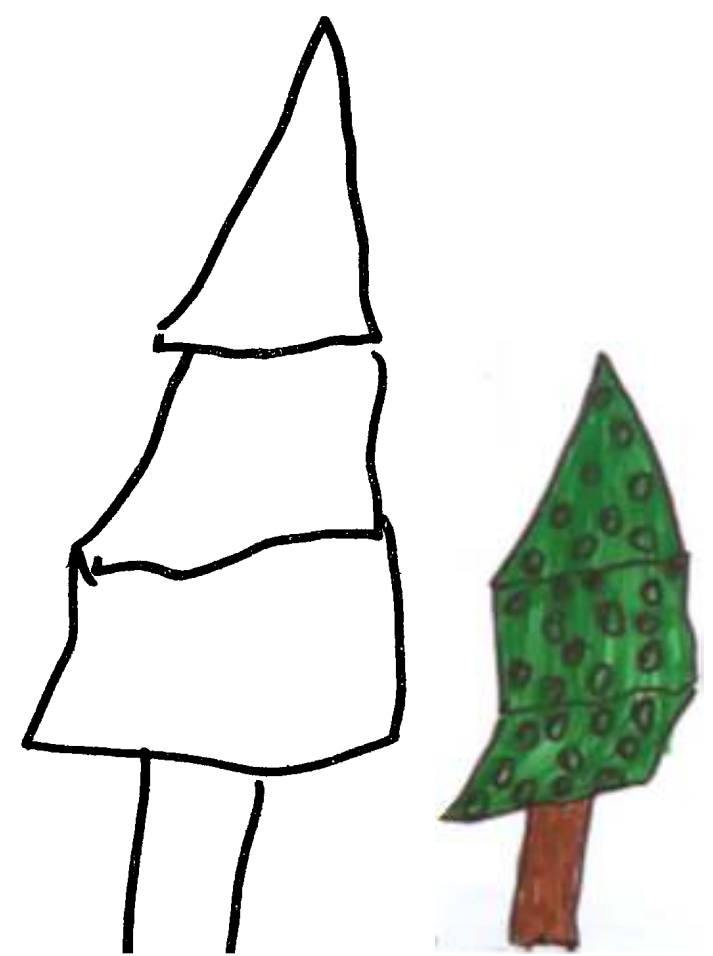

Figure 4 : dessins de sapins par des enfants Roms de 7 et 8 ans chant ;

Les musiciens intervenants ont également constaté que les EFIV, bien que pour la plupart de nationalité française, ne maîtrisaient pas bien la langue française. De plus, les EFIV ne connaissaient que peu, voire pas, de chansons enfantines françaises.

Les deux musiciennes intervenantes de chant ont alors créé des comptines à gestes pour essayer de sensibiliser des EFIV de 3 à 15 ans ayant divers parcours de scolarisation à la perception et de la production de phonèmes du français, ainsi qu'à la correspondance entre phonèmes et graphèmes $d u$ français. Les musiciennes intervenantes se sont réparties les tâches : la première a écrit les paroles des comptines en s'appuyant sur les phono-comptines de Basset ${ }^{4}$, la seconde s'est chargée des illustrations, à la fois de la comptine et du matériel didactique (à savoir, des dessins des gestes articulatoires oro-faciaux des phonèmes cibles, et des gestes pantomimiques représentant les gestes articulatoires oro-faciaux des phonèmes cibles).

L'utilisation des comptines à gestes créées a fait l'objet :

- d'une analyse qualitative par le biais :

- de l'observation du déroulement des séances par les deux musiciennes intervenantes de

- des retours des apprenants aux deux musiciennes intervenantes de chant ;

- de réunions hebdomadaires des musiciens de l'association intervenant sur l'aire d'accueil des « gens du voyage » de Geispolsheim.

- d'une analyse quantitative fondée sur :

- le nombre d'EFIV présents aux ateliers ;

- la durée de leur présence aux ateliers. 


\section{2. Propositions didactiques sur support de comptines à gestes} musiciennes intervenantes de chant de l'Association Ballade, comprennent pour chaque graphème étudié :

39

- le texte de la comptine décrivant le geste graphique cible (cf. Figure 5);

- l'illustration de la comptine décrivant le geste graphique cible (cf. Figure 6);

- l'illustration du geste articulatoire oro-facial du phonème cible (cf. Figure 7);

- l'illustration du geste pantomimique représentant le geste articulatoire oro-facial du phonème cible (cf. Figure 8 ).

40

Ces supports combinent ainsi les habiletés auditive, orale, proprioceptive, visuelle et kinésique. Ils ont été exploités dans une séance pédagogique détaillée ci-après.
Je suis le grand $\mathrm{A}$
Bonjour, je suis le grand A.
J'ai bien deux pieds, mais pas de bras.
Sur mes deux pieds, je tiens bien droit.
Dans une maison en forme de toit.
Dans certains mots, je viens trois fois.
Oui, par exemple dans : ananas.
Dans toutes les langues, je suis là.
En première place, l'alphabet m'a.
Et puis voilà, je suis le $\mathrm{A}$.

Figure 5 : texte de la comptine de BASSET décrivant le geste graphique $d u<A>$ tracé en capital

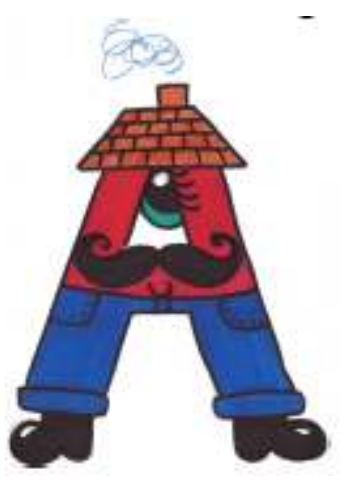

Figure 6 : illustration de la comptine décrivant le geste graphique $<A>$ tracé en capital
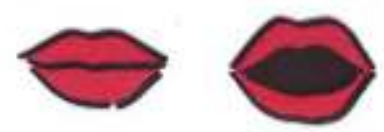

Figure 7 : illustration du geste articulatoire oro-facial du phonème /a/

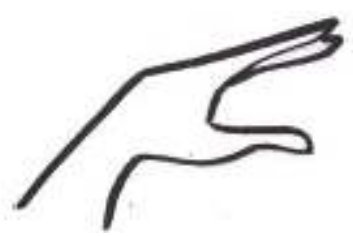

Figure 8 : illustration du geste pantomimique représentant le geste articulatoire oro-facial du phonème /a/ 
41 Le premier objectif de la séance est d'enseigner la production du phonème cible et d'expliciter les gestes articulatoires oro-faciaux correspondants. L'intervenant présente aux apprenants le phonème cible (par exemple /a/). Celui-ci est donné d'abord oralement et de manière isolée, puis oralement et en association avec le geste manuel pantomimique représentant le geste articulatoire oro-facial correspondant (cf. Figure 8). À chacune de ces deux étapes, les apprenants sont invités à répéter le support, qu'il soit oro-verbal et/ou gestuel. L'intervenant donne et explique le geste manuel pantomimique représentant les gestes articulatoires oro-faciaux requis pour la production $\mathrm{du}$ phonème cible. Pour les consonnes /s/ et /b/ par exemple, des informations en termes de lieu et de mode articulatoires apparaissent sur les illustrations, un tracé rouge pour la sifflante, des traits pour la plosive (cf. Figure 9).

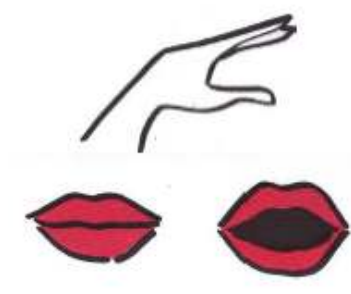

/a/

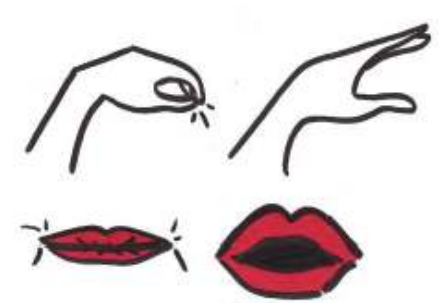

/b/

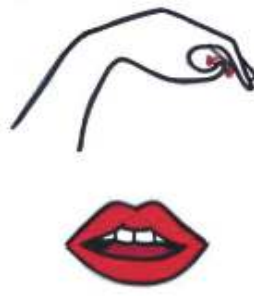

$/ \mathbf{s}$

Figure 9 : haut : illustrations des gestes pantomimiques représentant les gestes articulatoires orofaciaux cibles; bas : illustrations des gestes articulatoires oro-faciaux cibles graphème cible, ainsi que sa production. L'intervenant montre aux apprenants l'illustration de la comptine décrivant le geste graphique (cf. Figure 6). L'intervenant scande l'intégralité du texte de la comptine tout en pointant du doigt les parties du dessin concernées par l'énoncé. L'intérêt ici est de sensibiliser les apprenants à la forme graphique du phonème. L'intervenant demande ensuite aux apprenants de répéter après lui, énoncé après énoncé, le texte de la comptine, d'abord sans exploiter le geste de pointage des éléments repères sur l'illustration, puis avec. Cet exercice peut être réitéré avec l'enseignement/apprentissage du geste pantomimique représentant le geste graphique du graphème cible. Dans ce cas, le geste graphique peut être tracé dans l'espace ${ }^{5}$ ou sur support papier. Chaque énoncé peut être répété plusieurs fois si besoin, en groupes de taille variée ou individuellement selon l'objectif de l'intervenant et selon les besoins des apprenants.

Le troisième objectif de cette séance est de sensibiliser les apprenants au lien phonèmegraphème. Sur le principe du jeu «Pigeon vole", l'intervenant scande le texte de la comptine et les apprenants reçoivent pour consigne de produire le geste pantomimique représentant le geste articulatoire oro-facial étudié (cf. Figure 8) à chaque fois qu'ils discriminent perceptivement le phonème correspondant. L'intervenant affiche ensuite le texte de la comptine en format A3 et scande lentement le texte en pointant le graphème cible, toujours énoncé après énoncé. Ensuite, il reprend la lecture scandée et les apprenants tentent de discriminer visuellement le graphème cible et de produire le geste pantomimique du geste graphique à chaque fois qu'ils le reconnaissent. Les apprenants répètent ensuite eux-mêmes la comptine, énoncé après énoncé, en suivant la même consigne.

Le quatrième objectif de cette séance est la sensibilisation des apprenants à la prosodie. Il est possible de proposer le jeu du «Chef d'orchestre » : les apprenants sont installés 
en demi-cercle et l'intervenant ou un apprenant est placé face à eux de sorte d'être vu de tous. C'est à lui que revient la mission de donner le signal de départ et de fin du morceau, et de proposer des nuances de volume et de tempo. Ce jeu a par ailleurs l'avantage de faire travailler les niveaux segmental et suprasegmental en voix scandée, criée, parlée, chuchotée et murmurée; variété facilitant la découverte des différences acoustiques, perceptives et articulatoires conséquentes des modifications d'utilisation de l'appareil phonatoire. Pour étudier le langage suprasegmental, il est également intéressant d'exploiter l'activité "ritmimots" de Llorca (2008) puisqu'elle associe gestes rythmiques et parole. Les apprenants sont dans ce cas scindés en deux groupes, lesquels sont ensuite placés en face à face. Tour à tour, chaque groupe produit un énoncé de la comptine en battant la pulsation des mains. Ces activités permettent par ailleurs d'éviter un potentiel ennui résultant des répétitions, de maintenir l'attention et la concentration des apprenants, et de faciliter la mémorisation du texte de la comptine et par là, l'intégration phonologique.

\section{3. Analyse des effets de l'action pédagogique}

Du fait de la participation libre aux ateliers (comme expliqué auparavant), les données quantitatives des effets de l'utilisation de comptines à gestes n'ont pas pu être analysées. En effet, la présence et la durée de présence des EFIV dépendaient de nombreux facteurs, tels que :

- le beau temps, les EFIV étant alors souvent sollicités par leurs parents pour diverses tâches en dehors de l'aire ;

- les tâches ménagères, les EFIV, surtout les adolescentes, devant notamment aider au ménage, à la préparation des repas, à la garde des plus jeunes ;

- le travail, les EFIV, surtout les garçons adolescents, accompagnant les hommes.

En outre, peu d'EFIV restaient plus de trois semaines sur l'aire d'accueil des « gens du voyage » de Geispolsheim. De ce fait, l'organisation en séquence des enseignements/ apprentissages n'était pas possible car à chaque séance, le groupe d'EFIV présents n'était pas le même. Il n'était pas non plus envisageable de refaire chaque semaine la même séance étant donné que quelques EFIV étaient bien présents de la première à la dernière séance. L'action pédagogique présentée ci-avant (voir supra, chapitre 2. 2.) a ainsi été proposée lors de trois séances et pour trois phonèmes cibles $(/ \mathrm{a} /, / \mathrm{b} /$ et $/ \mathrm{s} /$ ).

L'analyse qualitative des effets de l'utilisation de comptines à gestes a montré que ces supports permettaient effectivement de sensibiliser les EFIV à l'enseignement/ apprentissage de la perception et de la production de phonèmes du français. Le dessin illustrant les paroles de la comptine a permis aux apprenants un accès immédiat au texte de la comptine. Ce support était particulièrement intéressant dans le contexte donné dans la mesure où les apprenants ne parlaient pas tous le français-en particulier ceux âgés de 3 à 6 ans -, et n'avaient pas les mêmes LM, et que les deux musiciennes intervenantes ne maîtrisaient aucune des LM des EFIV.

L'utilisation de gestes pantomimiques représentant les gestes articulatoires oro-faciaux des phonèmes cibles a apporté un aspect ludique à la comptine. En effet, les apprenants ont tous volontiers produit ces gestes. Ils ont également porté beaucoup d'intérêt à l'observation et à la production de leurs propres gestes oro-faciaux et de ceux des autres apprenants. Ainsi, certains exploraient de manière haptique leurs propres productions en mettant leurs mains devant et même dans leur bouche. L'utilisation de 
gestes pantomimiques représentant les gestes articulatoires oro-faciaux des phonèmes cibles a permis aux apprenants de se rendre compte que chaque phonème n'était pas produit de la même manière, et que les modifications de positionnement de langue, de lèvres et de mandibule, tout comme le mode d'échappement de l'air, et la vibration ou non des plis vocaux avaient un impact immédiat sur les sons produits.

L'utilisation de comptines à geste pour l'enseignement/apprentissage de la correspondance entre phonèmes et graphèmes du français a eu des effets mitigés. La majorité des apprenants produisait et mimait les gestes graphiques, et montrait avec fierté aux autres apprenants et aux musiciennes intervenantes leurs productions écrites (dessins et écrits) et mimées. Néanmoins, les apprenants les plus jeunes (parfois 3 ans) et les moins scolarisés ont été mis en difficulté par la production des gestes pantomimiques représentant les gestes graphiques. Il est aussi arrivé que des EFIV, prioritairement des adolescents de sexe masculin, quittent un atelier arguant qu'ils ne savaient ou ne souhaitaient pas écrire, et que des comptines portant sur ce thème ne les intéressaient pas.

51 Les musiciennes intervenantes ont également pu remarquer que la diversité des supports n'était pas suffisante. En effet, dès l'effet de nouveauté passé, les apprenants se désintéressaient très rapidement du matériel utilisé. Cependant, tous les EFIV voulaient pouvoir écrire ou dessiner.

Pour pallier les difficultés attentionnelles des EFIV, les musiciennes intervenantes ont intégré davantage de supports différents aux séances. Les comptines sur le thème des graphèmes ont été utilisées mais uniquement en introduction des séances, avec pour objectif pédagogique complémentaire l'échauffement des gestes oro-faciaux. À celles-ci ont été ajoutées des virelangues tels « Un chasseur sachant chasser sans son chien » ou "À la une dans la lune ». La séance continuait avec des chansons propres à diverses langues-cultures : "Vent frais » a par exemple été chantée en français, «Ederlezi » en romani, « Hajde Jano » en serbo-croate, ou encore "Shchedryk shchedryk » en ukrainien. Des chansons ont aussi été proposées en version plurilingues, comme «Vive le vent/ Jingle bells ». Pour faciliter l'accès au sens des chansons, des gestes pantomimiques étaient produits par les musiciennes intervenantes.

53 Afin d'augmenter le taux et la durée de présence des EFIV à chaque séance, et afin de développer leurs capacités attentionnelles, seuls ceux ayant participé à l'ensemble de la séance bénéficiaient de feutres et de feuilles pour dessiner et écrire pendant la dernière demie heure. En fin de séance était également mis à leur disposition un support consistant en un dessin et un mot le désignant en pointillés à compléter ( $c f$. Figure 10). Ce support était considéré par les apprenants comme très ludique et motivant, et s'est avéré utile par le développement de leurs compétences graphomotrices. Ainsi, ce support a permis de sensibiliser à l'écrit les EFIV par le biais du dessin.

54 Ce deuxième format de séance semble avoir été plus apprécié et plus efficient pour les apprentissages dans le contexte éducatif décrit. Le taux et la durée de fréquentation aux ateliers de chant ont augmenté, tout autant que l'attention des EFIV. 


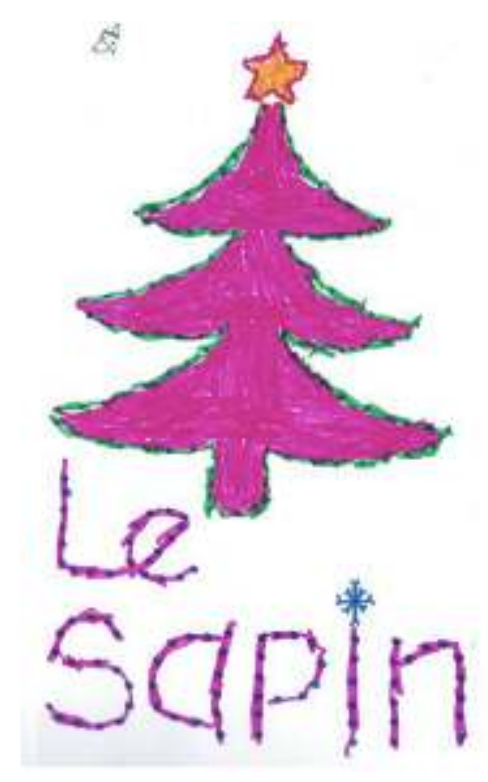

Figure 10 : dessin et mot en pointillés pour la chanson «Vive le vent/Jingle bells »

\section{Conclusion}

Cette recherche-action auprès d'EFIV a permis de tester l'intérêt de l'utilisation de comptines à gestes pour l'enseignement/apprentissage du FLES, notamment auprès de ce public dont les langues-cultures sont de transmission orale. De plus, utiliser des chansons extraites du folklore traditionnel français a permis de non seulement enseigner-apprendre la langue française, mais aussi une part importante de sa culture. Les aspects moteurs, rimiques, rythmiques et mélodiques des comptines à gestes rendent ce support particulièrement ludique, un atout des apprenants peu, voire pas scolarisés, et ayant ainsi peu l'habitude de solliciter leur attention dans un contexte éducatif de type classe.

L'apprentissage du français écrit apparaît fondamental pour l'inclusion des EFIV dans le système éducatif français et dans la société française. Pour ce faire, il est primordial de trouver des outils motivants pour les EFIV afin de les sensibiliser à l'écrit. L'envie d'apprendre le système de l'écriture est la condition sine qua non à leur motivation, mais il s'agit aussi de trouver des moyens ludiques et non-formels de développer leurs habiletés graphomotrices. Cela commence probablement par permettre à ce public à besoins spécifiques que sont les EFIV de pratiquer les jeux de mains et le dessin.

Ainsi, les aspects prosodiques, phonémiques et graphiques, mais aussi culturels du FLES peuvent être abordés de nombreuses manières. Cette recherche-action a mis en évidence que l'association de gestes manuels à différents types de voix et à des supports illustrés et orthographiés permet une approche ludique et un apprentissage réel.

Cette recherche indique que la comptine à geste peut être un support didactique intéressant pour l'enseignement/apprentissage de la perception et de la production de phonèmes du français. Ce support, notamment par son aspect multimodal qui permet d'aborder le langage dans ses dimensions verbale et non-verbale, peut également être utilisé pour enseigner-apprendre la relation entre phonème et graphème. 
Ce travail préliminaire pourrait être utilisé dans d'autres contextes d'enseignement/ apprentissage et avec d'autres phonèmes et graphèmes que ceux présentés au sein de cet article et en annexes. Les supports illustrés peuvent par ailleurs être simplifiés dans des séquences de cours ultérieures afin que les apprenants approchent progressivement les lettres sans ornement telles qu'ils les verront ensuite dans différentes situations authentiques.

L'exploitation des comptines à gestes extraites du folklore enfantin de la langue-culture cible permettrait de poursuivre ce travail pour sensibiliser les apprenants aux gestes emblématiques qui peuvent différer entre les langues-cultures à l'autre.

peuvent différer entre les langues-cultures à l'autre.

\section{BIBLIOGRAPHIE}

ALLEN L. Q., 1995, « The effects of emblematic gestures on the development and access of mental representations of French expressions ", The Modern Language Journal, $\mathrm{n}^{\circ}$ 79, p. 521-529.

ARLEO A. et DELALANDE J. (dir.), 2010, Cultures enfantines : universalité et diversité, Rennes, Presses Universitaires de Rennes, coll. « Le sens social ».

AUBIN E., 2008, La commune et les gens du voyage, $3^{\mathrm{e}}$ éd., Berger-Levrault, coll. « Le point sur ».

BADER, C. (2007). Yéniches. Les derniers nomades d'Europe. Paris, France : L'Harmattan.

BARA F., GENTAZ E. et COLÉ P., 2004, « Effet de l'exploration visuo-haptique et haptique de lettres dans les entraînements de préparation à la lecture », Approche Neuropsychologique des Apprentissages chez l'Enfant, n 78 , p. 189-194.

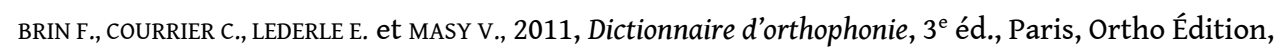
Paris, France.

CATROUX M., 2002, « Introduction à la recherche-action : modalités d'une démarche théorique centrée sur la pratique », dans M. HARDY et M. BOUGHEDAOUI (dir.), « La recherche-action : un autre regard sur nos pratiques pédagogiques ( $2^{\mathrm{e}}$ partie) », Cahiers de l'APLIUT, vo. 21, nº 3, p. 8-20. Disponible sur : <https://journals.openedition.org/apliut/4276>.

CAUSSADE D., CORNAZ S. et TISSERAND C., 2014, « La LSF pour apprendre le lexique d'une chanson en LE : une étude préliminaire », E-CRINI, $n^{\circ}$ 6, p. 1-27.

CLANET E., 2007, « La scolarisation des enfants du voyage au Centre national d'enseignement à distance », Distances et savoirs, vol. 4, $\mathrm{n}^{\circ} 5$, p. 559-574.

COLLETTA J.-M., 2004, Le développement de la parole chez l'enfant âgé de 6 à 11 ans : corps, langage et cognition, Sprimont, Mardaga.

CORNAZ S., 2014, L'apport de la voix chantée pour l'intégration phonético-phonologique d'une langue étrangère : application auprès d'italophones apprenants de FLE, thèse de doctorat en sciences du langage, soutenue à l'Université Grenoble Alpes. Disponible sur : <https://hal.archivesouvertes.fr/tel-01314777/document>. 
DUNOYER DE SEGONZAC M., 1991, Pour que vibre la Dynamique Naturelle de la Parole. Lyon, Éditions E. Robert.

FERREIRO E., GOMEZ PALACIO M., VERDENELLI M. et al., 1988, Lire-écrire à l'école, comment s'y apprennentils ?: Analyse des perturbations dans les processus d'apprentissage de la lecture et de l'écriture, Lyon, Canopé - CRDP de Lyon.

FEYEREISEN P. et HAVARD I., 1999, « Mental imagery and production of hand gestures while speaking on younger and older adults », Journal of nonverbal behavior, $\mathrm{n}^{\circ} 23, \mathrm{p} .153-171$.

FEYEREISEN P., BERREWAERTS J. et HUPET M., 2007, « Pragmatic skills in the early stages of Alzheimer's disease: an analysis by means of a referential communication task », International Journal of Language \& Communication Disorders, $\mathrm{n}^{\circ}$ 42, p. 1-17.

GILA-KOCHANOWSKI (DE) V., 1994, Parlons Tsigane. Histoire, culture et langue du peuple tsigane, Paris, L'Harmattan, coll. « Parlons ».

GUBERINA P., 1965, « La méthode audio-visuelle structuro-globale », Revue de phonétique Appliquée, $\mathrm{n}^{\circ} 1$, Mons, Université de Mons.

HUBBARD G., COOK A., TESTER S. et DOWNS M., 2002, « Beyond words: older people with dementia using and interpreting nonverbal behaviour ", Journal of Aging Studies, $\mathrm{n}^{\circ}$ 16, p. 155-167.

IVERSON J. M. et FAGAN M. K., 2004, « Infant Vocal-Motor Coordination: Precursor to the GestureSpeech System ? ", Child Development, vol. 75, n² 4, p. 1053-1066.

LIEGEOIS J.-P., 1994, Roma, Tsiganes, Voyageurs, Strasbourg, Conseil de l'Europe.

MARTIN L., 2011, Les représentations des enseignants sur les gens du voyage : quel impact sur la scolarisation de ce public ?, Mémoire de Master 2 Métiers de l'Enseignement de l'Éducation et de la Formation (spécialité : Enseignement du premier degré), Universités de Nantes, d'Angers et du Maine. Disponible sur : <https://dumas.ccsd.cnrs.fr/dumas-00781725/document>.

MCNEILL D., 1992, Hand and Mind: What gestures reveal about thought, Chicago, The University of Chicago Press.

-, 2000, Language and gesture, Cambridge, Cambridge University Press.

MIRANDA M. A., 2012, «L'intégration entre gestes et prosodie : une vision incarnée », Cahiers de linguistique française, $\mathrm{n}^{\circ}$ 30, p. 181-196.

MONTESSORI M., 1958, La découverte de l'enfant, pédagogie scientifique, Paris, Éditions Desclée De Brouwer, tome 1.

PLUCHON B., 2009, Les " gens du voyage », une catégorie problématique de l'action publique : analyse sociohistorique et ethnographique d'une catégorie, de ses enjeux et effets, thèse de doctorat en sociologie, soutenue à l'Université de Nantes.

RENARD R. (dir), 2002, Apprentissage d'une langue étrangère/seconde. 2. La phonétique verbo-tonale, Bruxelles, De Boeck Supérieur, coll. « Pédagogies en développement ».

SAUSSURE F., 1971 [1916], Cours de linguistique générale, Lausanne-Paris, Payot, coll. « Études et documents Payot ».

SERVICE GENS DU VOYAGE, 2017, Projet social 2017-2021. Disponible sur : < www.strasbourg.eu/ documents/976405/1521450/0/76320e95-1e86-602a-8aa8-f6b135e5662d $>$.

SUEYOSHI A. et HARDISON D. M., 2005, « The Role of Gestures and Facial Cues in Second Language Listening Comprehension ", Language Learning, vol. 55, nº 4, p. 661-699. 
TELLIER M., 2006, L'impact du geste pédagogique sur l'enseignement/apprentissage des langues étrangères : Étude sur des enfants de 5 ans, thèse de doctorat en Linguistique, soutenue à l'Université ParisDiderot.

-, 2009, « The development of gesture », in K. DE BOT et R. W. SCHRAUF (dir.), Language Development Over the Lifespan, New York / Oxon, Routledge, p. 191-216.

THIBAULT C., 1999, De la distinction tsigane : identité et religion chez les voyageurs de Rennes, Mémoire d'anthropologie, soutenue à l'Université Laval. Disponible sur : <https:// www.collectionscanada.gc.ca/obj/s4/f2/dsk2/ftp01/MQ42022.pdf>.

WELSCHINGER R. L. V., 2007, Les Jenischs d'Alsace : approche d'une culture nomade marginale, thèse de doctorat en ethnologie, soutenue à l'Université de Strasbourg.

-, 2013, Vanniers (Yeniches) d'Alsace. Nomades blonds du Ried, Paris, L'Harmattan.

\section{ANNEXES}

ANNEXES : supports pédagogiques imprimables à l'échelle
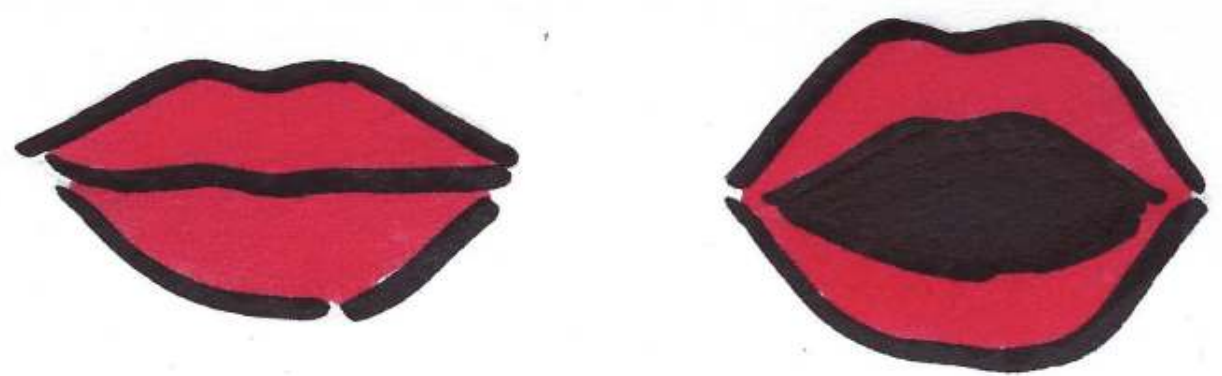

Support 1 : illustration de YEZHKOVA du geste articulatoire oro-facial du phonème /a/

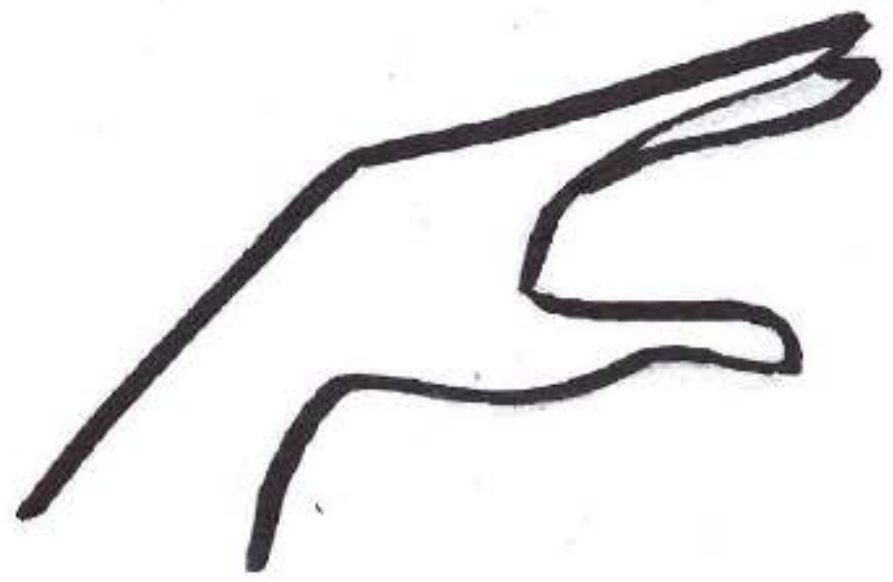

Support 2 : illustration de YEZHKOVA du geste pantomimique du geste articulatoire oro-facial du phonème $/ \mathrm{a} /$

Je suis le grand A

Bonjour, je suis le grand A.

J'ai bien deux pieds, mais pas de bras.

Sur mes deux pieds, je tiens bien droit. 
Dans une maison en forme de toit.

Dans certains mots, je viens trois fois.

Oui, par exemple dans : ananas.

Dans toutes les langues, je suis là.

En première place, l'alphabet m'a.

Et puis voilà, je suis le A.

Support 3 : comptine de BASSET décrivant le geste graphique $\mathrm{du}<\mathrm{A}>$ majuscule

Je suis le petit a

Bonjour, je suis le petit a.

J'ai une tête toute arrondie

Posée sur mon oreiller.

Les yeux fermés,

Je fais semblant d'être endormi

Car j'ai envie de rester au lit.

Support 4 : comptine de CAUSSADE décrivant le geste graphique $\mathrm{du}<\mathrm{a}>$ minuscule

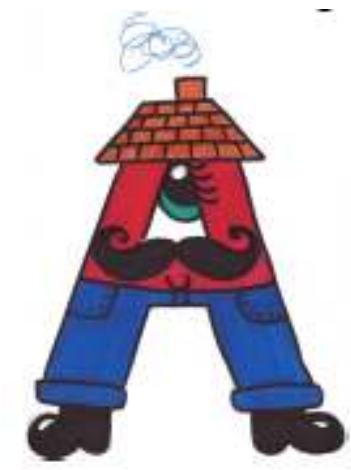

Support 5 : illustration de YEZHKOVA de la comptine décrivant le geste graphique du $<$ A $>$ majuscule 


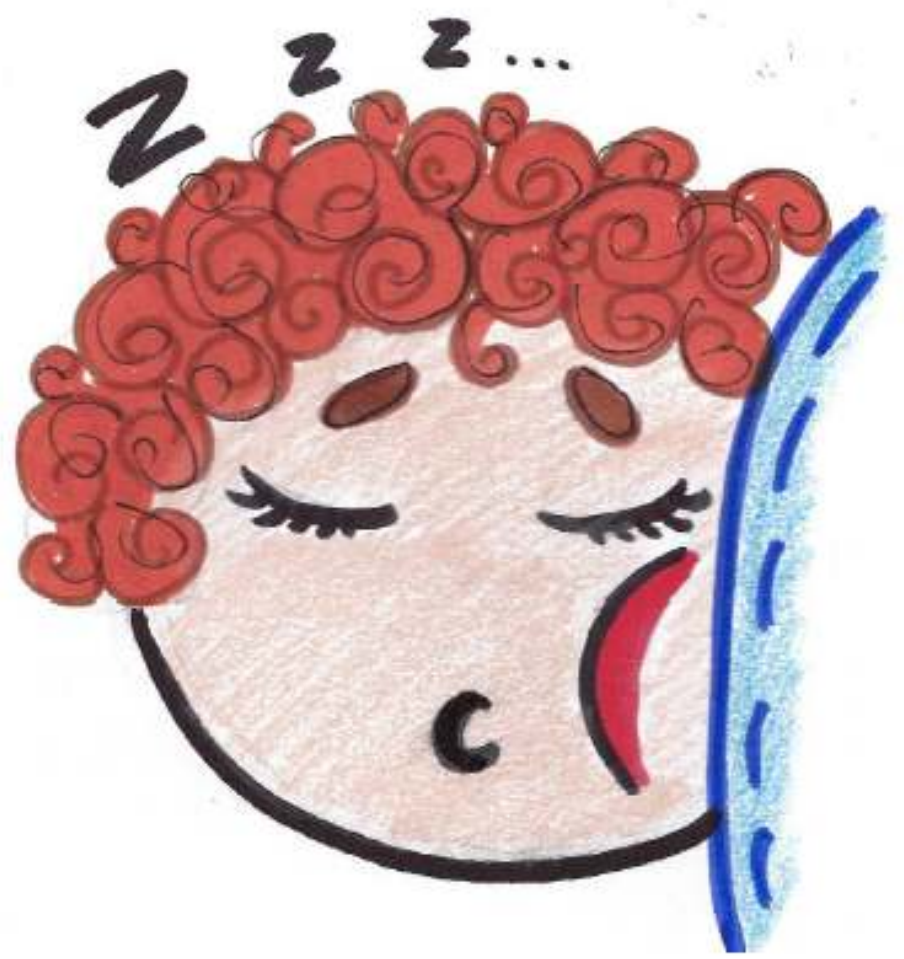

Support 6 : illustration de YEZHKOVA de la comptine décrivant le geste graphique du $<\mathrm{a}>$ minuscule
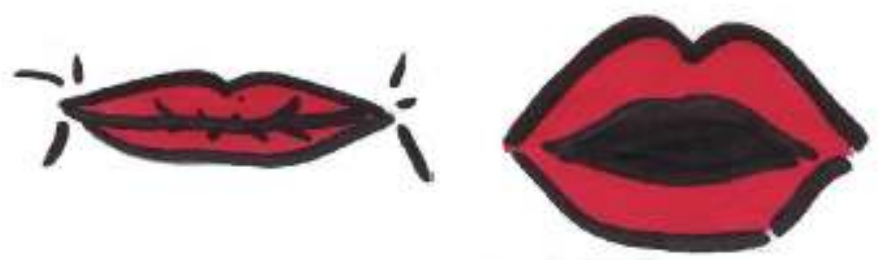

Support 7 : illustration de YEZHKOVA du geste articulatoire oro-facial du phonème /b/

Papa B et maman $b$

Mon papa est le grand B,

Celui qui est tout bombé

Comme les joues de bébé.

Ma maman est l'autre $b$

Dont seul le ventre est bombé

Parce qu'elle attend un bébé

Support 9 : comptine de CAUSSADE décrivant le geste graphique $d u<B>$ majuscule et du $<\mathrm{b}>$ minuscule 
Enseignement/apprentissage du FLES aux EFIV : propositions didactiques de l'u...

20

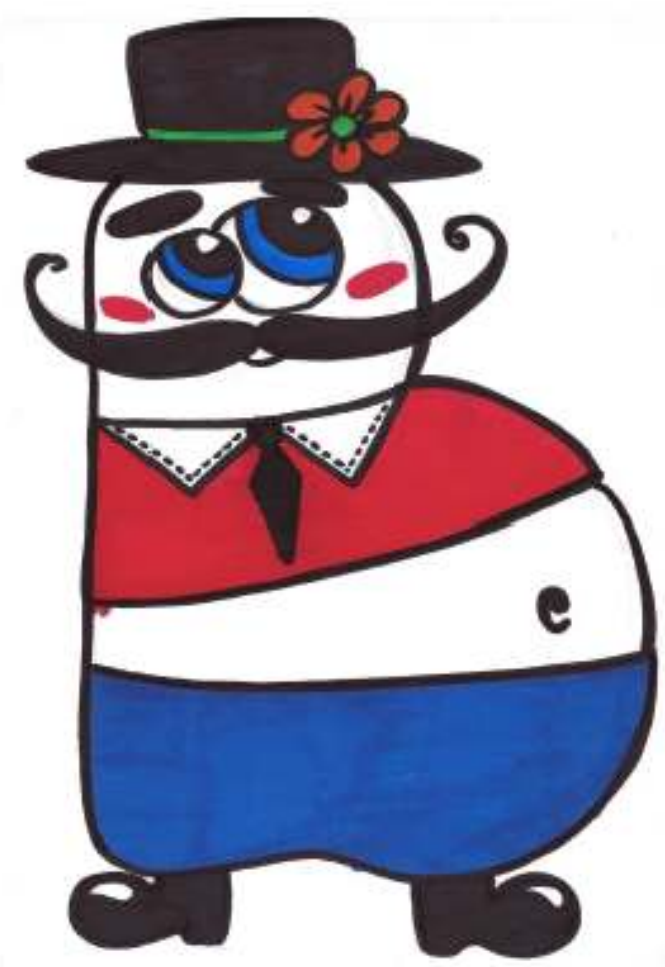

Support 10 : illustration de YEZHKOVA de la comptine décrivant le geste graphique du $<\mathrm{B}>$ majuscule

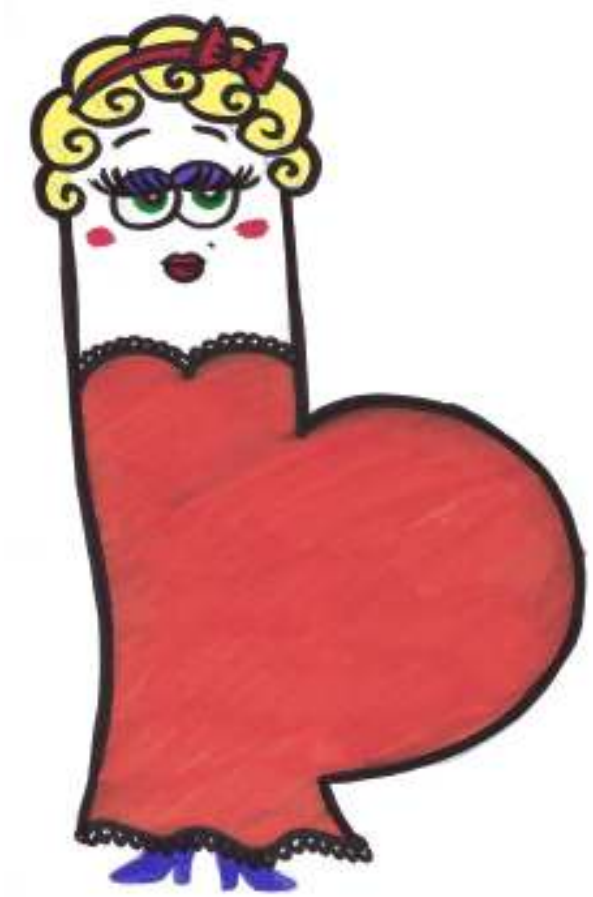

Support 11 : illustration de YEZHKOVA de la comptine décrivant le geste graphique du $<\mathrm{b}>$ minuscule

Corela, HS-30 | 2020 


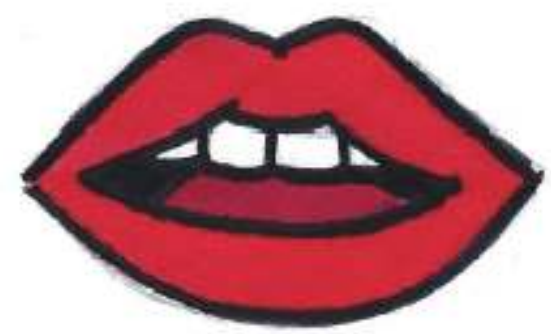

Support 12 : illustration de YEZHKOVA du geste articulatoire oro-facial du phonème /s/ Croissants

Le grand $C$ est un croissant de lune.

Et le petit $c$ est un croissant

Qui ressemble à la lune.

Moi celui que je préfère croquer

C'est le petit c.

Support 14 : comptine de CAUSSADE décrivant le geste graphique $\mathrm{du}<\mathrm{C}>$ majuscule et $\mathrm{du}<\mathrm{c}>$ minuscule

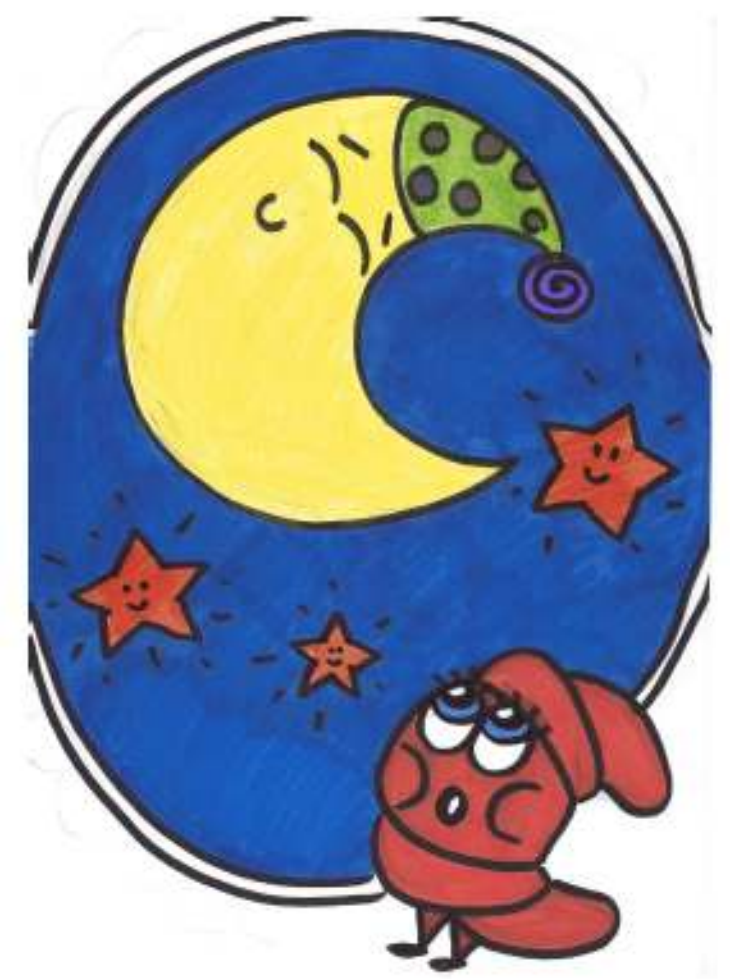

Support 15 : illustration de YEZHKOVA de la comptine décrivant le geste graphique du $<\mathrm{C}>$ majuscule et $<\mathrm{C}>$ minuscule 


\section{NOTES}

1. La recherche-action est définie par Catroux (2002:8) comme un «processus destiné à doter tous les participants de la scène éducative [...] des moyens d'améliorer leurs pratiques grâce à leurs expériences éclairées et nourries des savoirs théoriques en cours ".

2. McNeill (1992) catégorise les gestes communicatifs comme suit: les signes (gestes codifiés composant les langues signées); les emblèmes (gestes produits conjointement ou non à la parole et dotés d'un sens conventionnel dépendant d'une langue-culture donnée); les pantomimes (gestes de mime ou d'imitation produits en l'absence de parole) ; les gesticulations (i.e. gestes coverbaux) (gestes produits de manière spontanée conjointement à la parole, que McNeill (ibid.) distingue en quatre types : les déictiques ou gestes de pointage ; les iconiques qui représentent un référent concret comme un objet, une action ou une disposition spatiale ; les métaphoriques qui représentent un référent abstrait ; les battements qui mettent en exergue une syllabe ou bien un mot).

3. La traduction n'étant pas toujours possible quand l'intervenant ne maîtrise pas la/les langue(s) des apprenants. D'autant plus que le principe de la traduction n'est pas toujours fonctionnel quand le groupe d'apprenants ne possède pas les mêmes langues maternelles.

4. Disponibles sur : <http://www.phonemus.fr/phonocomptines/phonocomptines.htm>.

5. L'intervenant doit veiller au risque de tracé inversé s'il fait face aux apprenants.

\section{RÉSUMÉS}

Pour les enfants issus de familles itinérantes et de voyageurs (EFIV), le français est une langueculture seconde, voire étrangère. Devant l'analphabétisme et l'illettrisme de masse, ainsi que le faible taux de scolarisation des EFIV, l'insertion socioculturelle des communautés désignées sous l'appellation administrative de "gens du voyage» est primordiale. Les langues-cultures des " gens du voyage » étant principalement de transmission orale, l'utilisation de gestes manuels et de comptines à gestes comme supports de l'enseignement/apprentissage du français semble particulièrement pertinent. Un autre argument en faveur de l'utilisation de ces supports auprès d'EFIV est que la communication non-verbale fait partie intégrante du langage et qu'elle favoriserait la compréhension et la production langagière, notamment pour l'apprentissage de la prosodie et de la phonologie. Au vu de ces éléments, des comptines à gestes ont été créées afin de permettre l'enseignement/apprentissage de la perception et de la production des phonèmes du français, ainsi que la correspondance entre phonèmes et graphèmes. Ces supports pédagogiques ont été testés au sein de l'association Ballade auprès d'EFIV, et leur impact a été analysé afin de permettre une réévaluation des propositions didactiques.

For children from the Traveler communities, French is a second language-culture or even a foreign one. Given the high rate of illiteracy, and the low rate of schooling among children from the Traveler communities, the sociocultural insertion of people from the Traveler communities is of central importance. The languages-cultures of the Traveler communities being mainly orally transmitted, the use of gestures and gesture-play songs as materials of teaching-learning of French seems especially relevant. Another argument in favour of the use of those materials with children from the Traveler communities is that non-verbal communication is an integral part of language and would support language understanding and production, notably to learn prosody 
and phonology. In view of those elements, gesture-play songs have been created to teach-learn French phonemes perception and production, and correspondence between phonemes and graphemes. This pedagogical material has been tested with children from the Traveler communities by the association Ballade. Their impact has been tested in order to revaluate the didactic proposals.

\section{INDEX}

Mots-clés : perception, production, phonèmes, graphèmes, FLES, EFIV, comptine à gestes, langue-culture, multimodalité, enseignement/apprentissage.

Keywords : perception, production, phonemes, graphemes, FLES, gesture-play song, languageculture, multimodality, teaching/learning.

\section{AUTEURS}

\section{DIANE CAUSSADE}

LISEC, Université de Haute-Alsace

\section{SANDRA CORNAZ}

LESLA, Université Lyon 2

\section{MARINA YEZHKOVA}

Association Ballade 